\title{
Comparative Study of the Effect of Laparoscopic and Open Surgery on Acute Cholecystitis
}

\author{
Jian Lin* \\ The Second People’s Hospital of Shifang City, Shifang, Sichuan 618000, China
}

\begin{abstract}
Objective: To compare the clinical effect of laparoscopic and open surgery on acute cholecystitis. Method: clinical data of 200 cases of acute cholecystitis patients in our hospital from July 2010 to July 2014 were divided into laparoscopic cholecystectomy (LC) group and open cholecystectomy(OC)group. Comparison was made from various aspects to show the feasibility of laparoscopic cholecystectomy of acute cholecystitis. Results: the operation time, off-bed activity time, postoperative gastrointestinal function recovery time and hospital stay time of LC group were all lower than OC group $(t=2.785, t=2.825, t=2.831, t=2.904, p$ $<0.05)$; OC group's postoperative complications was $23.0 \%$, higher than that $(9.0 \%)$ of LC $\operatorname{group}\left(\chi^{2}=3.764, p<0.05\right)$. Conclusion: Under the chosen strict condition of surgical indications and delicate surgery operation, the application of laparoscopic cholecystectomy in the treatment of acute cholecystitis is safe and feasible.
\end{abstract}

\author{
KEYWORDS \\ Acute cholecystitis \\ Cholecystectomy \\ Laparoscope
}

\section{Introduction}

Acute cholecystitis is one of acute abdominal pains, with acute congestion and edema of gallbladder wall as the main pathological basis and right hypochondrium pain, tenderness, nausea, and vomiting as the main clinical manifestations. The preferred treatment is cholecystectomy. The current cholecystectomy in clinical mainly include open cholecystectomy and laparoscopic cholecystectomy. Both the surgical treatments will result in different clinical effect. Clinical data of 200 cases of acute cholecystitis patients in our hospital from July 2010 to July 2014 were

\footnotetext{
Copyright $\odot 2015$ Jian Lin

doi: $10.18686 /$ aem.v4i3.8

Received: August 1, 2015; Accepted: September 2, 2015; Published online: September 19, 2015

This is an open-access article distributed under the terms of the Creative Commons Attribution Unported License (http://creativecommons.org/ licenses/by-nc/4.0/), which permits unrestricted use, distribution, and reproduction in any medium, provided the original work is properly cited.

${ }^{\star}$ Corresponding author: The Second People's Hospital of Shifang city, Shifang, Sichuan 618000, China. E-mail: lijian888@sina.com
}

divided into LC group and OC group. Comparison was made from various aspects to show the feasibility of laparoscopic cholecystectomy of acute cholecystitis.

\section{Data and method}

\subsection{General information}

200 cases of acute cholecystitis patients, who received acute cholecystitis surgery in the general surgery department of our hospital from July 2010 to July 2014 , were selected. Based on the surgery, patients were divided into OC group and LC group, 100 cases in each group. The differences of basic information between two groups had no statistical significance, with comparability $(p>0.05)$. OC group consisted of 45 males and 56 females; average age was (65.4 $\pm 10.6)$ years old; onset time was $(36 \pm 22)$ hours; gallbladder ultrasound thickness was $(4.0 \pm 2.0)$ $\mathrm{mm}$. LC group consisted 55 males and 46 females; average age was $(63.8 \pm 11.2)$ years old; onset time was $(34 \pm 36)$ hours; gallbladder ultrasound thickness was $(4.1 \pm 2.3) \mathrm{mm}$. 
Table 1. Comparison of perioperative indexes between two groups $(\bar{x}+s)$.

\begin{tabular}{cccccc}
\hline Group & Case & Operation time(min) & $\begin{array}{c}\text { Postoperative gastrointestinal } \\
\text { function recovery time(d) }\end{array}$ & $\begin{array}{c}\text { Hospital stay time (d) } \\
\text { Off-bed activity time } \\
(\mathrm{h})\end{array}$ \\
\hline LC group & 100 & $75.9 \pm 41.6$ & $1.6 \pm 0.6$ & $4.8 \pm 1.7$ & $11.2 \pm 6.6$ \\
OC group & 100 & $109.4 \pm 31.2$ & $2.9 \pm 0.6$ & $9.6 \pm 2.9$ & $24.5 \pm 7.8$ \\
$t$ & $/$ & 2.785 & 2.825 & 2.831 & 2.904 \\
$P$ & $/$ & $<0.05$ & $<0.05$ & $<0.05$ & $<0.05$ \\
\hline
\end{tabular}

\subsection{Inclusion criteria and exclusion criteria 2.2.1. Inclusion criteria}

Patients with cholecystitis diagnosis meeting the diagnostic criteria listed in Diagnosis and Treatment Guide of Acute Biliary Infection and surgical indication, all patients' onset time did not surpass 72 hours.

\subsubsection{Exclusion criteria}

Patients with other acute abdominal disease which requires surgical treatment; patients whose surgery may lead to cardiac and renal insufficiency; patients with mental illness or their immediate family members has a history of mental illness; patients with surgery contraindication, onset time of over 72 hours.

\subsection{Method}

Patients from both groups used endotracheal intubation general anesthesia. OC group patients were subjected to right costal margin incision into the abdomen, received gallbladder excision by combination of anterograde resection and retrograde resection. The LC group used standard four holes method to conduct laparoscopic cholecystectomy, that was to select under the belly button for A hole, xiphoid process for B hole, right under the costal margin for $\mathrm{C}$ and $\mathrm{D}$ holes. This was good for a clearer operative field exposure, shortening the operation time, $\mathrm{CO}_{2}$ pneumoperitoneum pressure was $12-15 \mathrm{mmHg}$. High head and lower feet was about $10^{\circ}-15^{\circ}$, left side slope of $10^{\circ}$; the dissection of gallbladder triangle was carefully done to free gallbladder artery, which was closed and cut off by double titanium clip. Upon confirmation, the cystic duct was isolated and closed at the part closely to the gallbladder ampulla by titanium clip. The vast majority received anterograde resection, combined with retrograde excision if necessary, electric coagulation was made on the errhysis or bleeding parts to reduce the heat burns on the liver. Drainage tube was placed in the lesser omentum foramen and drawn out from the right axillary front line holes. Then removed the gallbladder. And completed the surgery. The operation time, blood loss, off bed activity time, recovery time of gastrointestinal function, postoperative complications and average hospital stay time of patients in two groups were observed [1].

\subsection{Observation index}

All patients' intra-operative and postoperative situations were observed and compared. Intra-operative condition included operative time and intraoperative blood loss, postoperative condition included postoperative exhaust time, postoperative off bed time, drainage flow and hospital stay time. The occurrence of complications due to surgery of patients in two groups during hospitalization was analyzed and compared.

\subsection{Statistical analysis}

SPSS 19.0 was used to analyze, the measurement data was expressed as $(\overline{\mathrm{x}} \pm \mathrm{s})$ and analyzed by $\mathrm{t}$ test, count data was expressed as a percentage and analyzed by $\mathrm{x}^{2}$ test, $p<0.05$, there was statistical significance.

\section{Result}

\subsection{Comparison of perioperative indexes between two groups}

Table 1 showed that the operation time, postoperative gastrointestinal function recovery time, hospital stay time and off-bed activity time of LC group were lower than OC group, and the difference was statistically significant $(t=$ $2.785, t=2.825, t=2.831, t=2.904,(p<0.05))$.

\subsection{Comparison of postoperative complications between two groups}

Table 2 showed that OC group's occurrence rate of postoperative complications was $22.0 \%$, higher than that of LC group (8.00\%), and the difference was statistically $\operatorname{significant}\left(\chi^{2}=3.764, p<0.05\right)$.

Table 2. Comparison of complications between two groups.

\begin{tabular}{ccc}
\hline Complication & OC group $(n=100)$ & LC group $(n=100)$ \\
\hline Bleeding & 5 & 2 \\
Bile leakage & 4 & 2 \\
Pulmonary infection & 3 & 2 \\
Abdominal infection & 4 & 2 \\
Poor wound healing & 6 & 0 \\
Total & 22 & 8 \\
\hline
\end{tabular}




\section{Discussion}

Open cholecystectomy is a common clinical method of biliary tract surgery, but it has disadvantages of large incision, unfit for postoperative recovery and large damage to patients. The patients with old age or poor condition who used the operation method often requires careful assessment of body state before surgery. Now, with the development of the endoscopic technology and the wide application of laparoscopic surgery, laparoscopic cholecystectomy obtains wide application. In comparison to the traditional open cholecystectomy, laparoscopic cholecystectomy has advantages of small incision, small trauma and good for postoperative recovery, thus it is also suitable for old age or poor condition patients. But some clinical observation showed that the common bile duct injury risk of laparoscopic treatment is higher than the traditional surgery. This often makes some patients convert to laparotomy after treatment due to difficult to control. So, for the patients with laparoscopic surgical treatment, the surgeon should closely observe the condition of abdominal viscera, identify the junction part of gallbladder ampulla and cystic duct and expose the gallbladder triangle as far as possible during operation. The clinical observation showed that the effect of laparoscopic cholecystectomy on acute cholecystitis is obviously superior to open cholecystectomy. In this study, the LC group's operation time, postoperative gastrointestinal function recovery time, hospital stay time, off bed activity time were lower than OC group. On the other hand, as the LC was a minimally invasive treatment, patients' postoperative complications after LC treatment were significantly lower than the OC patients, patients had fewer complications, so their postoperative recovery was fast and the hospital stay time was shortened. Therefore, for patients with acute cholecystitis, LC has great advantage than OC. However, the LC should pay attention to the following issues:

(1) LC should strictly grasp the indications: patients with acute calculous cholecystitis disease of less than 72 hours; patients whose condition was not relieved or even aggravated through conservative treatment 24 to 48 hours after onset. Patients with simple acute calculous cholecystitis by preoperative examination. For the above situation, flexible treatment should be conducted, if anatomy is not clear, surgery is difficult, in order to ensure safety, we can transfer to open laparotomy. LC should be avoided if acute calculous cholecystitis course is more than 3 days, and without any formal treatment, preoperative check suspect gangrenous cholecystitis or gallbladder perforation [2].

(2) If marked hyperemia, edema of gallbladder wall, gallbladder empyema, high gallbladder pressure, gallbladder clamping difficulties occur during surgery due to incarcerated calculus in cystic duct, gall bladder decompression should be made by fundus of gallbladder puncture first, then pliers can be used to lift up the bottom of the gallbladder or the gall bladder neck traction and pull outwards to show the Calot triangle $\mathrm{T}$ structure, we also can appropriately expand the separation scope of gallbladder triangle. When finding the enlarged "ductus cysticus", don't blindly clamp and cut off. Upon clear identification of the relationship of common bile-duct cystic duct, occlude cystic duct, if using absorbable clip, the occlusion is more reliable.

(3) Must be familiar with the anatomy of the bile duct and have the resolution capability of anatomical variation. Remember not to cut off the pipeline in the separation process, some gallbladder triangle anatomy are not sure, the cystic duct can be "clipped without cut off", removing the gallbladder retrograde, and then deal with cystic duct and its blood vessels [3].

(4) For gallbladder triangle area, cold blunt separation should be mainly used and electrocoagulation heat separation is complementary to avoid bile duct electric burns. These measures can effectively reduce the incidence of iatrogenic bile duct injury, reduce the occurrence of postoperative complications and reduce the being converted to open laparotomy rate.

(5) After LC, there are more surgical trauma and inflammatory exudation for patients with acute calculous cholecystitis, we should routinely place abdominal cavity drainage tube to help to perform postoperative observation of whether patients have bleeding or bile leakage, so as to treat timely. On the other hand, in case of bile leakage, the abdominal cavity drainage can also provide some help for treatment. This study analyzed the therapeutic effect of two kinds of surgical treatment on acute cholecystitis. The result showed that two groups' intra-operative time had no statistically significance, while intra-operative blood loss in LC group was obviously lower than OC group. Thus, the injury of laparoscopic cholecystectomy is obviously smaller than open cholecystectomy. At the same time, the postoperative analysis showed that LC group had lower exhaust time, postoperative off bed time, flow rate and hospital stay time than the OC group. It can be seen the postoperative recovery of patients undergoing laparoscopic surgery is superior to the patients with laparotomy therapy. This study also compared two groups of patients' surgeryrelated complications, the result showed that the difference of surgery-related complications between two groups was statistically significant, the LC group was significantly lower than OC group. It can also be seen that laparoscopic treatment also can reduce surgery-related complications while reducing intraoperatie injury and being beneficial to patients' postoperative recovery $[4,5]$.

In conclusion, the surgical treatment of patients with acute cholecystitis should strictly master operative indication, be accurate and skilled, if there is difficulty for excision, we should transfer to laparotomy decisively. 


\section{References:}

1. Yu AJ, Zhang XJ, Wu SY, et al. Logistic analysis of acute cholecystitis laparoscopic cholecystectomy transfer laparotomy's risk factors. Shandong Medical Journal. 2011;51(8):62-63.

2. Zhang ZQ, Rong DQ, Dong Q, et al. Comparison analysis of acute cholecystitis laparoscopic and open operation. Journal of Clinical Hepatology. 2010;26(1):71-72.

3. Yang YQ, Zhou W, Lin MQ, et al. Laparoscopic gallbladder excision of 312 cases of acute cholecystitis. Journal of hepatopancreatobiliary surgery. 2010;22(3):213-215.

4. Song JN, Luo YZ, Hong M, et al. Clinical analysis of 130 cases of laparoscopic treatment of acute cholecystitis. Chongqing medicine. 2010;33(5):6, 553-554.

5. Sun HX, Wang YM, Deng YJ, et al. Laparoscopic cholecystectomy for acute cholecystitis. Chinese journal of Clinical Medicine. 2010;(6):845-846. 